Article original

\title{
Étude des facteurs pronostiques de survenue de grossesse chez les couples infertiles pris en charge en dehors de la fécondation in vitro par injection intra-cytoplasmique de spermatozoide dans l'Ouest Algérien
}

\author{
Study of the prognostic factors of pregnancy in infertile couples managed outside in \\ vitro fertilization by intra-cytoplasmic sperm injection in western Algeria
}

\author{
Nadjet Belhachemi1, Faten Hakim1, Karima Chenni2, Djamila Larbi2, Adnane Adelouahab2, Belkacem Chafi1 \\ 1Service de gynécologie obstétrique. Etablissement Hospitalier et Universitaire 1er Novembre, Oran. \\ 2 Service de Biostatistique. Faculté de Médecine d'Oran.
}

Article reçu le 18 mai 2018 / Accepté le 02 juin 2018

\section{MOTS CLÉS \\ Couple infertile, Fac- teurs pronostiques, Age de la femme et de l'homme, Tabagisme passif, Infertilité pri- maire et secondaire, Oli- go-asthéno-tératosper- mie sévère, Grossesse}

\begin{abstract}
Résumé
Introduction - L'Assistance Médicale à la Procréation permet la prise en charge des couples infertiles par de nombreuses techniques : injection intra- cytoplasme de spermatozoïde, fécondation in vitro et insémination intra-utérine. Cependant le traitement des couples infertiles ne se limite pas à l'Assistance Médicale à la Procréation. La proportion de grossesses naturelles est loin d'être négligeableavec des moyens plus simples. Ce mode d'obtention de grossesse dépend des facteurs pronostiques de l'infertilité. Ces facteurs sont représentés par l'âge de la femme et à l'âge de l'homme, le tabagisme passif, la durée et le type de l'infertilitéainsi que lescauses de l'infertilité. Cette étude a été menée afin d'identifier les facteurs pronostiques de survenue de grossesse chez les couples infertiles pris en charge en dehors de la fécondation in vitro ou injection intra-cytoplasme de spermatozoïde.
\end{abstract}

Matériels et méthodes - C'est une étude prospective, mono centrique s'étendant sur 4 ans de janvier 2009 à décembre 2012.L'étude des facteurs pronostiques de survenue de grossesse s'est faite sur des comparaisons statistiques entre deux groupes : un groupe ayant fait une grossesse $(n=147)$ et un groupe n'ayant pas réussi à concevoir $(n=458)$. Une analyse multivariée par régression logistique binaire a été réalisée afin d'identifier les facteurs pronostiques.

Résultats - L'étude a colligé 605 couples. Le nombre de grossesses obtenues était de 147 (24,3\%).L'analyse multivariée a retenu les facteurs pronostiques suivants: l'âge de la femme supérieur à 35 ans et celui de l'homme supérieur à 45 ans, le tabagisme passif chez la femme, l'infertilité primaire, une durée d'infertilité

Auteur correspondant: inesse06@hotmail.com

JFMO : N4, Juin 201829 
supérieure à 3 ans, ainsi que l'oligo-asthéno-tératospermie sévère.

Conclusion - Les facteurs de survenue d'une grossesse sont intimement liés aux facteurs pronostiques de l'infertilité. L'âge de la femme et de l'homme, le type, la durée et la cause de l'infertilité, sont déterminants du pourcentage de réussite et du délai de survenue de la grossesse en dehors de la fécondation in vitro ou injection-intra cytoplasme de spermatozoïde.

\section{KEY WORDS}

Infertile couple, Prognostic factors, Age of women and men, Passive smoking Primary and secondary infertility, Severeoligo-astheno-terato-spermia, Pregnancy.

\section{Introduction}

L'infertilité est définie par l'Organisation Mondiale de la Santé (OMS) comme l'échec de survenue d'une grossesse clinique après 12 mois de rapports sexuels non protégés [1] et après six mois si la femme a plus de 35 ans [2].Dans le cadre de la santé reproductive, tout être humain à droit à la procréation et l'OMS considère actuellement l'infertilité comme une pathologie à part entière[3].L'absence de démarrage de grossesse chez le couple quand il décide d'en avoir et que ce désir ne se réalise pas, est source de frustration. Cette impatience va le conduire à une consultation du médecin généraliste ou spécialiste et une demande de médicalisation.

L'Assistance Médicale à la Procréation (AMP) permet la prise en charge des couples infertiles par de nombreuses techniques : injection intra-cytoplasme de spermatozoïde (ICSI), fécondation in vitro (FIV)et insémination intra-utérine (IIU). Cependant, le traitement des couples infertiles ne se limite pas à l'AMP. Avec des moyens plus simples, la proportion de grossesses naturelles est loin d'être négligeable.

En effet, ce mode d'obtention de grossesse dépend des facteurs pronostiques de l'infertilité pour une prise en charge spécifique. Les facteurs pronostiques rapportés dans la littérature sont représentés par le type primaire ou secondaire, la durée d'infertilité, l'âge de la femme et à un degré moindre celui de l'homme, le tabagisme passif ainsi que les facteurs étiologiques de l'infertilité. 
L'âge de la femme est un facteur déterminant de la fertilité. En effet, la fertilité chez les femmes diminue lorsque l'âge augmente, avec une accentuation nette de ce déclin à partir de 35 ans [4]. L'infertilité n'est plus l'apanage de la femme, comme c'est le cas dans notre société où elle est considérée la première responsable de ce problème. L'enquête épidémiologique de Thonneau en 1991, a permis de mettre en évidence la responsabilité partagée de l'homme et de la femme dans l'infertilité du couple [5]. L'infertilité chez l'homme est rapidement apparue comme un facteur important, représentant $50 \%$ des causes des difficultés à concevoir [6]. Il a été observé qu'une proportion élevée d'hommes jeunes avait des caractéristiques spermatiques défavorables [7], avec des histocomptabilités glaire - spermatozoiide détériorées [8].

En Algérie, peu d'études ont été publiées sur le couple infertile. Cette étude a été menée afin d'analyser les facteurs pronostiques de survenue de grossesse chez les couples infertiles pris en charge en dehors de FIV/ICSI et constitue de ce fait la première étude en Algérie.

\section{Matériels et méthodes}

\section{Type et population d'étude}

Il s'agit d'une étude analytique prospective, réalisée au service de gynécologie obstétrique de l'établissement hospitalier et universitaire (EHU) 1er Novembre-Oran, durant la période allant de janvier 2009 à décembre 2012.

Ont été exclus de l'étude les couples qui ont refusé de faire leur suivi conjointement au sein de l'unité, les couples dont les femmes étaient âgées de plus de 45 ans et ceux n'ayant pas de papiers administratifs confirmant leur mariage en cas d'indication d'insémination intra-utérine.

\section{Protocole d'étude}

Le recueil des données et le suivi ont été faits au fur et à mesure du recrutement exhaustif des couples. L'enquête a été réalisée au moyen d'un questionnaire qui était constitué de 5 volets :

- le premier se basait sur l'identification du couple (âge, profession, niveau socio-économique et lieu de résidence)

- le deuxième portait sur l'histoire de l'infertilité (type et durée de l'infertilité)

- le troisième précisait les examens cliniques et paracliniques chez la femme et chez l'homme qui ont permis d'orienter vers une origine de l'infertilité (féminine, masculine, mixte ou idiopathique) et d'identifier les causes féminines et/ou masculines

- le quatrième s'intéressait à la thérapeutique reçue par le couple infertile et le suivi qui s'est étendu sur une durée de 3 à 5 ans de leur inclusion jusqu'à l'arrêt de traitements ou survenue de grossesse avec la naissance d'enfant.

- le cinquième concernait la survenue de grossesse et son évolution.

Les données ont été recueillies à travers un entretien avec le couple retraçant leur histoire d'infertilité et leurs parcours diagnostique et thérapeutique. Après un interrogatoire minutieux, un examen clinique a été réalisé séparément chez la femme et son conjoint.

Les patientes sont convoquées le 2ème jour du cycle pour un bilan biologique standard, un bilan hormonal de base et une échographie endovaginale.

Elles sont ensuite revues au 12ème jour du cycle pour effectuer une hystérosalpingographie, un monitorage folliculaire par échographie et un test post-coital.

Le bilan chez l'homme est fait d'un spermogramme et d'un spermocytogramme voire spemoculture si présence de signes infectieux. Il est orienté ensuite chez un andrologue pour un suivi régulier tous les 3 mois.

Un diagnostic étiologique et une orientation thérapeutique ont été établis sur la base du bilan clinique et paraclinique. Un calendrier de suivi a été réalisé chez les couples tous les 2 à 3 mois en fonction de la durée d'infertilité et de l'âge de la femme.

Des examens de contrôle ont été réalisés chez la femme (bilans biologiques tous les 3 à 6 mois, bilans hormonaux avec sérologie tous les ans et échographies pelviennes à J2 et J12 du cycle). Chez l'homme, un contrôle systématique du spermogramme à 3 mois d'intervalle du premier puis tous les 3 à 6 mois avec une sérologie tous les ans ont été effectués.

Le consentement éclairé signé a été obtenu auprès des couples.

\section{Variables de l'étude}

Les facteurs pronostiques étudiés sont représentés par :

- L'âge de la femme est classé en moins et plus de 35 ans et l'âge de l'homme en moins et plus de 45 ans.

- Le niveau socio- économique : les indicateurs couramment utilisés pour mesurer le statut social sont le niveau d'éducation, la profession et le revenu. Trois niveaux ont été identifiés : le niveau élevé regroupait le couple qui travaillait avec un bon revenu et un niveau intellectuel élevé, le niveau moyen regroupait au moins un membre du couple qui travaillait et un niveau intellectuel moyen, le niveau bas regroupait le couple non salarié n'ayant aucun niveau d'instruction.

- Le tabagisme passif chez la femme dont le mari est fumeur.

- L'indice de masse corporelle (IMC = Poids $(\mathrm{Kg}) /(\operatorname{taille2}(\mathrm{m}))$ pour les adultes selon la classification de l'OMS[9].

- L'infertilité est évaluée selon sa durée : $\leq 2$ ans, 3-5 ans, $>5$ ans.

- Le type d'infertilité est classé en primaire et secondaire. 
L'infécondité est dite aprimaire» si le couple n'a jamais conçu. Elle est dite «secondaire» si une grossesse est survenue quelque soit son évolution (fausse couche, grossesse extra-utérine (GEU) ou interruption volontaire de grossesse (IVG)) [10].

- L'origine de l'infertilité est classée en féminine, masculine, mixte ou inexpliquée. L'origine féminine de l'infertilité est répartie en causes ovulatoire, tubaire ou les deux associées. L'origine masculine est repartie selon les anomalies du spermogramme interprété selon les normes de l'OMS 2010 en :

- spermogramme normal : la numération $\geq 15$ millions $/ \mathrm{ml}$, la mobilitéa $+b>30 \%$ et la forme typique selon la classification de Kruger $\geq 4 \%$,

- asthénospermie $(a+b<30 \%)$,

- tératospermie (forme typique $<4 \%$ ),

- oligo-asthéno-tératospermie (OAT) : modérée (numération de 5 - 15 millions $/ \mathrm{ml}$, mobilité : $10<a+b<30 \%$ et forme typique au moins $4 \%$ ) ousévère (nombre de $1-5$ millions $/ \mathrm{ml}$, mobilité $a+b<10 \%$ et forme typique moins de $4 \%$ ).L'OAT est dite très sévère lorsque le nombre de spermatozoïdesest inférieur à 1 million par $\mathrm{ml}$, lorsqu'ils ne sontpas ou très peu mobiles, et lorsque les formes anormales sont très nombreuses)[11].

\section{Analyse statistique}

L'étude des facteurs pronostiques de survenue ou non de grossesse hors FIV/ICSI a été réalisée par la comparaison de deux groupes le groupe ayant fait une grossesse $(n=147)$ confirmé par un dosage biologique de B HCG positif et par une échographie endovaginale, quelque soit l'évolution de celle-ci; et le groupe n'ayant pas réussi à concevoir $(n=$
458).

L'analyse statistique des données a été réalisée à l'aide du logiciel SPSS 20.0. Deux tests d'association paramétriques ont été utilisés : le $\square 2$ pour rechercher la relation entre deux variables qualitatives et le test de l'écart réduit pour la comparaison des moyennes.

Afin d'identifier les facteurs pronostiques de survenue de grossesse, une analyse par régression logistique binaire a été réalisée en choisissant comme variable dépendante " survenue de grossesse». Le modèle initial incluait l'ensemble des facteurs statistiquement liés à la grossesse à un seuil de $20 \%$.La sélection des variables restant dans le modèle final a été faite selon une stratégie pas à pas descendante et les facteurs significatifs ont été retenus à un seuil de $5 \%$.

\section{Résultats}

Parmi les 760 couples infertiles ayant bénéficié d'une prise en charge au cours de cette période, $605(79,6 \%)$ ont été inclus dans cette étude. Les 155 couples restant $(20,4 \%)$ ont été exclus car ils nécessitaient d'emblée FIV/ICSI voire une adoption. Les causes d'exclusion sont multiples :

- $87(11,4 \%)$ femmes présentaient des obstructions tubaires bilatérales,

- $40(5,3 \%)$ hommes avaient une azoospermie ou une oligo-asthéno-tératospermie très sévère.

- $28(3,7 \%)$ femmes présentaient une réserve ovarienne effondrée ( $\mathrm{FSH}>20$ et une hormone antimüllérienne (AMH) inférieure à $0,2 \mathrm{ng} / \mathrm{ml}$ ).

Les caractéristiques de la population d'étude sont résumées dans le tableau 1.

Tableau 1. Caractéristiques de la population d'étude

\begin{tabular}{|c|c|c|}
\hline Paramètres d'infertilité & Effectif $(n)$ & Effectif (\%) \\
\hline \multicolumn{3}{|l|}{ Age moyen (ans) } \\
\hline Femme & $32,6 \pm 5,8(\min 18-\max 45)$ & \\
\hline Homme & $39,4 \pm 7,5(\min 24-\max 70)$ & \\
\hline \multicolumn{3}{|l|}{ Niveau socio- économique } \\
\hline Bon & 92 & 15,2 \\
\hline Moyen & 439 & 72,6 \\
\hline Bas & 74 & 12,2 \\
\hline \multicolumn{3}{|l|}{ Tabagisme chez les hommes } \\
\hline \multicolumn{3}{|l|}{ Tabagisme passif chez les femmes } \\
\hline Absent & 451 & 74 \\
\hline Présent & 158 & 26 \\
\hline \multicolumn{3}{|l|}{ IMC moyen $\left(\mathrm{kg} / \mathrm{m}^{2}\right)$} \\
\hline Femme & $26,4 \pm 4,3(\min 15.6-\max 45.3)$ & \\
\hline Homme & $26 \pm 2,2(\min 18,6-\max 37,3)$ & \\
\hline Durée moyenne d'infertilité(ans) & $4,8 \pm 3,4(\min 1-\max 25)$ & \\
\hline
\end{tabular}




\begin{tabular}{lll}
\hline $\begin{array}{l}\text { Type d'infertilité } \\
\text { Primaire }\end{array}$ & 445 & 73.6 \\
$\quad$ Secondaire & 160 & 26.4 \\
Origine de l'infertilité & & \\
$\quad$ Féminine & 135 & 22,3 \\
Masculine & 208 & 34,4 \\
Mixte & 161 & 26,6 \\
Inexpliquée & 101 & 16,7
\end{tabular}

Le nombre de grossesses obtenues après prise en charge était de 147 grossesses soit $24,3 \%$.

Etude des facteurs pronostiques de l'infertilité selon la survenue ou non de grossesse hors FIVIICSI

L'étude de survenue de grossesse a été réalisée selon les facteurs pronostiques suivants: l'âge de la femme et de l'homme, le niveau socio- économique, le tabagisme passif chez la femme, l'IMC chez la femme ainsi que la durée, type et origine de l'infertilité.

- Comparaison des caractéristiques des couples infertiles dans les deux groupes (tableau 2)Les femmes ayant conçu étaient significativement plus jeunes que les femmes n'ayant pas réussi à concevoir $(p<0,001)$. Le même résultat a été obtenu chez les hommes $(p<0,001)$.

Il n'existe pas d'association significative entre le niveau socio-économique et la survenue de grossesse, mais il existe une différence significative entre le tabagisme passif chez la femme et la survenue de grossesse avec un taux de $16,2 \%$ versus $27,1 \%(p=0,007)$. Il n'a pas été démontré d'association statistiquement significative entre l'IMC chez la femme et la survenue de grossesse.

La durée moyenne d'infertilité chez les couples qui ont réussi à concevoir était significativement inférieure par rapport à ceux qui n'ont pas obtenu de grossesse $(p<0,001)$. Le nombre de grossesses en infertilité secondaire $(30,7 \%)$ était significativement supérieur par rapport à l'infertilité primaire $(22 \%)$ avec un $\mathrm{p}<0,001$.

Le nombre le plus important de grossesses a été obtenu lorsque l'origine de l'infertilité était féminine ou inexpliquée respectivement à $29,2 \%$ et $25,6 \%$.Il existe une différence significative entre les deux groupes quant à l'origine masculine de l'infertilité $(p=0,023)$.

Tableau 2. Caractéristiques des couples infertiles avec ou sans survenue de grossesses.

\begin{tabular}{|c|c|c|c|}
\hline Paramètres d'infertilité & $\begin{array}{l}\text { Présence de Grossesse } \\
\qquad n=147\end{array}$ & $\begin{array}{l}\text { Absence de Grossesse } \\
\qquad n=458\end{array}$ & $P$ \\
\hline \multicolumn{4}{|l|}{ Age (ans) } \\
\hline \multicolumn{4}{|l|}{ Femme } \\
\hline Moyenne & $30.9 \pm 5.6(\min 20, \max 43)$ & $33.2 \pm 5.8(\min 18, \max 45)$ & 0.000 \\
\hline Groupes & & & 0,046 \\
\hline$\leq 35$ & $113(26,8)$ & $304(73,2)$ & \\
\hline$>35$ & $34(18,4)$ & $154(81,6)$ & \\
\hline \multicolumn{4}{|l|}{ Homme } \\
\hline Moyenne & $36.8 \pm 6.9(\min 25, \max 70)$ & $40.2 \pm 7.4(\min 24, \max 68)$ & 0.000 \\
\hline Groupes & & & 0,02 \\
\hline$\leq 45$ & $129(27,2)$ & $354(72,8)$ & \\
\hline$>45$ & $18(14,8)$ & $104(85,2)$ & \\
\hline Niveau socio- économique & & & NS \\
\hline Bon & $23(25,3)$ & $68(74,7)$ & \\
\hline Moyen & $108(24,5)$ & $333(75,5)$ & \\
\hline Bas & $15(22,1)$ & $53(77,9)$ & \\
\hline Tabagisme passif & & & 0,007 \\
\hline Absent & $122(27,1)$ & $329(72,9)$ & \\
\hline
\end{tabular}




\begin{tabular}{|c|c|c|c|}
\hline Présent & $25(16,2)$ & $129(83,8)$ & \\
\hline IMC femme & & & NS \\
\hline$<18$ & $1(10)$ & $9(90)$ & \\
\hline $18-24,9$ & $57(25,9)$ & $163(74,1)$ & \\
\hline $25-29,9$ & 61 & $182(74,9)$ & \\
\hline$\geq 30$ & $27(22,9)$ & $91(77,1)$ & \\
\hline \multicolumn{4}{|l|}{ Durée d'inferilité (ans) } \\
\hline Moyenne & $3.5 \pm 2.7(\min 1, \max 14)$ & $5.2 \pm 4(\min 1, \max 25)$ & 0.0007 \\
\hline Groupes & & & 0,000 \\
\hline$\leq 2$ & $69(37,1)$ & $117(62,9)$ & \\
\hline $3-5$ & $54(22,8)$ & $183(77,2)$ & \\
\hline$>5$ & $24(13,2)$ & $158(86,8)$ & \\
\hline Type d'Infertilité & & & 0.001 \\
\hline Primaire & $93(20,9)$ & $352(79,1)$ & \\
\hline Secondaire & $54(33,8)$ & $106(66,2)$ & \\
\hline \multicolumn{4}{|l|}{ Origine de l'infertilité } \\
\hline Feminine & & & NS \\
\hline Absente & $94(24,7)$ & $287(75,3)$ & \\
\hline Tubaire & $22(23,9)$ & $70(76,1)$ & \\
\hline Ovulatoire & $27(23,9)$ & $86(76,1)$ & \\
\hline Tubaire et Ovulatoire & $4(21,1)$ & $15(78,9)$ & \\
\hline Masculine & & & 0,023 \\
\hline Spermogramme normal & $70(28,3)$ & $177(71,7)$ & \\
\hline OAT modéré & $27(26,2)$ & $76(73,8)$ & \\
\hline OAT sévère & $16(12,9)$ & $108(87,1)$ & \\
\hline Asthenospermie & $27(26,2)$ & $76(73,8)$ & \\
\hline Teratospermie & $7(25)$ & $21(75)$ & \\
\hline Inexpliquée & $24(23,8)$ & $77(76,2)$ & NS \\
\hline
\end{tabular}

Analyse univariée et multivariée de survenue de la grossesse selon les facteurs pronostiques.

- Analyse univariée

L'analyse univariée a retenu six co-variables diminuant les chances de grossesses : l'âge supérieur à 35 ans chez les femmes et supérieur à 45 ans chez les hommes, le tabagisme passif, une durée d'infertilité supérieure à 3 ans, l'infertilité primaire, et l'OAT sévère (tableau 3 ).

Tableau 3. Analyse univariée à $20 \%$ des facteurs pronostiques de la survenue de grossesse.

\begin{tabular}{|c|c|c|c|}
\hline Variables avec $p<20 \%$ & OR & [IC $95 \%]$ & $\mathbf{P}$ \\
\hline \multicolumn{4}{|l|}{ Age de la femme } \\
\hline$\leq 35$ & 1 & & \\
\hline$>35$ & 0.47 & {$[0.30-0.73]$} & $<0.001$ \\
\hline \multicolumn{4}{|l|}{ Age de l'homme } \\
\hline$\leq 45$ & 1 & & \\
\hline$>45$ & 0.41 & {$[0.23-0.73]$} & $<0.002$ \\
\hline \multicolumn{4}{|l|}{ Niveau socio-économique } \\
\hline Bas & 0.83 & & NS \\
\hline Moyen & 0.95 & & NS \\
\hline Bon & 1 & & \\
\hline
\end{tabular}




\begin{tabular}{|c|c|c|c|}
\hline \multicolumn{4}{|l|}{ Tabagisme passif } \\
\hline Absent & 1 & & \\
\hline Présent & 0.52 & {$[0.32-0.84]$} & $<0.005$ \\
\hline \multicolumn{4}{|l|}{ IMC de la femme } \\
\hline$<18$ & & & NS \\
\hline $18-24.9$ & 1 & & NS \\
\hline $25-29.9$ & & & NS \\
\hline$\geq 30$ & & & NS \\
\hline \multicolumn{4}{|l|}{ Durée d'infertilité } \\
\hline$\leq 2$ & 1 & & \\
\hline $3-5$ & 0.5 & {$[0.32-0.76]$} & $<0.001$ \\
\hline$>5$ & 0.25 & [0.15- 0.43$]$ & \\
\hline \multicolumn{4}{|l|}{ Type d'infertilité } \\
\hline Secondaire & 1 & & \\
\hline Primaire & 0.51 & [0.34- 0.77] & $<0.001$ \\
\hline \multicolumn{4}{|l|}{ Origine de l'infertilité } \\
\hline \multicolumn{4}{|l|}{ Féminine } \\
\hline Absente & 1 & & NS \\
\hline \multicolumn{4}{|l|}{ Tubaire } \\
\hline \multicolumn{4}{|l|}{ Ovulatoire } \\
\hline \multicolumn{4}{|l|}{ Tubaire et Ovulat-oire } \\
\hline \multicolumn{4}{|l|}{ Masculine } \\
\hline Spermogramme Normal & 1 & & \\
\hline \multicolumn{4}{|l|}{ OAT Modérée } \\
\hline OAT Sévère & 0.37 & {$[0.20-0.67]$} & $<0.001$ \\
\hline \multicolumn{4}{|l|}{ Asthénospermie } \\
\hline \multicolumn{4}{|l|}{ Tératospermie } \\
\hline Inexpliquée & 1 & & NS \\
\hline
\end{tabular}

\section{Analyse faite sur des valeurs valides}

NS (non significatif), OR (Odds Ratio), IC95\% (Intervalle de confiance à 95\%)

Ces facteurs ont été introduits dans des modèles de régression logistique multiple avec une probabilité à $5 \%$.

- Analyse multivariée

L'analyse multivariée a retenu six variables significative- ment associées à l'infertilité dans le modèle final : l'âge supérieur à 35 ans chez les femmes et supérieur à 45 ans chez les hommes, le tabagisme passif, une durée d'infertilité supérieure à 3 ans, l'infertilité primaire, et l'OAT sévère (tableau 4).

Tableau 4. Modèle de régression logistique binaire sur la probabilité de survenue de grossesse chez les couples infertiles $(a=5 \%)$

\begin{tabular}{|c|c|c|c|}
\hline Variables avec $p<5 \%$ & OR ajusté & [IC $95 \%]$ & $\mathbf{P}$ \\
\hline \multicolumn{4}{|l|}{ Age de la femme } \\
\hline$\leq 35$ & 1 & & \\
\hline$>35$ & 0.54 & [0.33-0.89] & 0.017 \\
\hline \multicolumn{4}{|l|}{ Age de l'homme } \\
\hline$\leq 45$ & 1 & & \\
\hline$>45$ & 0.46 & {$[0.24-0.87]$} & 0.018 \\
\hline
\end{tabular}




\begin{tabular}{|c|c|c|c|}
\hline Absent & 1 & & \\
\hline Présent & 0.51 & {$[0.31-0.85]$} & 0.010 \\
\hline \multicolumn{4}{|l|}{ Durée d'infertilité (ans) } \\
\hline$\leq 2$ & 1 & & \\
\hline $3-5$ & 0.51 & {$[0.32-0.80]$} & 0.004 \\
\hline$>5$ & 0.30 & {$[0.17-0.53]$} & $10^{-3}$ \\
\hline \multicolumn{4}{|l|}{ Type d'infertilité } \\
\hline Secondaire & 1 & & \\
\hline Primaire & 0.56 & {$[0.36-0.88]$} & 0.012 \\
\hline \multicolumn{4}{|l|}{ Origine masculine } \\
\hline Spermogramme Normal & 1 & & \\
\hline OAT Modérée & 1.045 & {$[0.60-1.82]$} & NS \\
\hline OAT Sévère & 0.41 & {$[0.22-0.76]$} & 0.005 \\
\hline Asthénospermie & 1.15 & {$[0.65-2.03]$} & NS \\
\hline
\end{tabular}

NS (non significatif), OR (Odds Ratio), IC95\% (Intervalle de confiance à 95\%)

\section{Discussion}

Dans cette étude, la survenue de grossesse a été analysée par rapport à différents facteurs d'infertilité. Il a été constaté que plusieurs facteurs semblent déterminants dans la survenue de grossesse : l'âge de la femme et l'âge de l'homme, le tabagisme passif chez la femme ainsi que la durée, le type et l'OAT sévère dans l'origine masculine de l'infertilité.

Toutes les études s'accordent que l'âge de la femme constitue un facteur pronostique majeur et déterminant en termes de fertilité naturelle ou d'AMP. De nombreuses études ont établi la valeur seuil d'âge féminin à 35 ans à partir de laquelle la fertilité chez la femme chute de manière significative $[12,13,14]$. Cette diminution de la fertilité est liée à la diminution de la qualité ovocytaire et donc embryonnaire, comme l'atteste l'augmentation des avortements spontanées (40\% après 42 ans) et les anomalies chromosomiques [15]. Par contre ledéclindelafertilitédeshommesliée àl'âge,commecausedudéclinglobaldelafertilité, reste un sujet de controverse.Certaines études basées sur la reproduction assistée n'ont trouvé aucun effet significatif de l'âge paternel sur la fécondabilité $[16,17]$. Alors que d'autres études ont montré une diminution du taux de grossesses cliniques avec l'âge paternel à partir de 40 ans [18] ou de 45 ans [19].

Actuellement, l'âge de l'homme est de plus en plus incriminé dans la baisse de la fertilité. Si l'âge de déclin de la fertilité chez la femme est bien connu, le seuil de cette baisse chez l'homme reste difficile à préciser. "Si les hommes conservent leur potentiel de fertilité plus longuement que les femmes, il est cependant important de rappeler que la paternité tardive est de plus en plus associée à divers types de maladies congénitales et à des avortements » [19].

Les effets délétères du tabagisme sur la santé sont bien connus, mais le tabagisme peut affecter également la fertilité. UneétudeHollandaise[20] et une autre anglaise [21] ont montré l'influence négative du mode de vie (tabac, alcool, café, thé, IMC)sur la diminution des chances et l'allongement du délai de conception naturelle. De même qu'Augood dans une méta-analyse a montré qu'en FIV, les femmes fumeuses présentaient une réduction de la fécondité avec un $O R$ de 0,66 (IC à 95\% 0,49 à 0,88) par rapport aux non-fumeuses [22].

L'impact du tabagisme actif sur la fertilité de la femme est bien connu mais qu'on est-il du tabagisme passif chez la femme? Peu d'études se sont intéressées au tabagisme passif. Hull. Ma constaté qu'un retard de conception, statistiquement significatif, supérieur à 6mois,estobservéchezlesfemmesprésentantuntabagismeactifoupassif.Ce retard à la conception s'accroît avec l'importance de la consommation tabagique [23].

L'effet propre du tabac a été plus étudié, et un rapport a été publié en 2006 par la Société Américaine pour la Médecine de la Reproduction (SARM) [24].

Peu d'études se sont intéressées à évaluer le taux de survenue de grossesse par rapport à la durée d'infertilité. L'étude de Matalliotakis qui a examiné les données de 297 femmes entreprenant un traitementpar FIV, rapporte que les patientes enceintes ont une durée d'infertilité plus courte que celles n'obtenant pas de grossesse (3 versus 3.5 ans) [25] alors que Lintsen et al.dans leur première étude nationale, n'ont pas trouvé d'influence de la durée d'infertilité sur les résultats de FIV [20]. Par contre, dans la deuxième étude ils constatent que chaque année supplémentaire d'infertilité diminue les chances de grossesses évolutives [26]. Donc plus la durée d'infertilité augmente, plus les chances de grossesses diminuent.

En effet, l'infertilité primaire diminueles chances de grossesse par rapport à l'infertilité secondaire. La même constatation a été faite par l'étude deBotchanen 2001portant sur6139cyclesd'insémination artificielle avec sperme de donneurs [27] et Linsten en 2005, au Pays Basqui a mené une étude nationale chez les femmes sur les chances de grossesses dans la première année de leur traitement FIV [20]. Enfin,Shibaharaet al. 
observe que la notion de grossesse, ou mieux d'accouchement antérieur, augmente les chances de grossesse lors d'un cycle de FIV [28].

Plusieurs auteurs ont constaté que l'infertilité d'origine masculine s'accompagnait plus souvent d'échecs d'implantation en FIV que les autres causes d'infertilité, dans une population d'hommes de moins de 35 ans [29,30]. D'autres ont constaté que l'infertilité masculine sévère restait inefficace face à la FIV avec des taux de fécondation chutant de $72 \%$ à $18 \%$ [31].

Une relation étroite entre le taux de grossesse et les différents paramètres spermatiques a été démontrée par Bostofte $E$ et al. qui constate que le taux de grossesse ainsi que la chance d'avoir un enfant $(4.5 \%$ vs $21.3 \%)$ chute avec la diminution de la numération ( $>80 \%$ vs $<20 \%$ ) et la morphologie des spermatozoïdes (tératospermie importante) [32]. Alors que Jouannet $P$ et al. retrouve que le taux de grossesse augmentait avec la mobilité spermatique mais diminuait significativement dès que la numération est au-dessous de 5106 spermatozoïdes/ $\mathrm{ml}$ [33].

Ces études confirment que le taux de survenue de grossesses est en corrélation étroite avec le nombre, la morphologie et la mobilité des spermatozoïdes et c'est l'altération de ces trois paramètres "OAT sévère » qui offre au couple le moins de chances pour concevoir.

\section{Les limites de l'étude}

Certains points méritent d'être soulignés. Le niveau socio- économique limité des couples infertiles a joué un rôle dans nos prescriptions et sur nos conduites thérapeutiques. De même que le nombre restreint des tentatives d'I IU intraconjugale a fait que les chances de grossesses restaient diminuées.

\section{Conclusion}

Les facteurs de survenue d'une grossesse sont intimement liés aux facteurs pronostiques de l'infertilité. Ces facteurs pronostiques sont déterminants du pourcentage de réussite et du délai de survenue de la grossesse en dehors de FIV/ICSI.

L'information des couples sur les mécanismes de la reproduction humaine est donc indispensable et un élément primordial de la prise en charge.Une hygiène de vie saine en particulier l'arrêt du tabac et la lutte contre le tabagisme passif font partie intégrante du traitement.

Cette évaluation permet de définir la population qui nécessite un réel recours à des interventions biologiques et médicales pour ces pathologies et de réelles possibilités de prévention et de traitement.

Conflits d'intérêt : Les auteurs ne déclarent aucun conflit d'intérêt.

Remerciements : Nous tenons à remercier l'ensemble des couples qui ont participé à cette étude.

\section{Références bibliographiques}

[1] Zegers- hochshild F, Adamsan G.D, de Mouzon J et al. International committee for monitoring assisted reproductive technology (ICMART) and WHO revised glossary of ART terminology. Fertil Steril 2009.

[2] Cooper T.G, Noonan E, von Eckardstein S. World health organization reference values for human semen characteristics. Hum reproduct update 2010,16 (3) : 231-245.

[3] Rapport de l'Institut national de la sante et de la recherche médicale et par l'Agence de la biomédecine. Troubles de la fertilité, état des connaissances et pistes pour la recherche. 2011.

[4] Thonneau P. Les paternités tardives. CNGOF. Extrait des Mises à jour en Gynécologie Médicale. Andrologie 2008.

[5] Thonneau P, Marchand S, Tallec A, et al. Incidence et causes principales de l'infertilité chez une population résidente (1.850.000) de trois régions françaises (1988-1989). Hum Reprod1991, 6 (6): 811.

[6] Boivin J, Bunting L, Collins J.A, et al. International estimates of infertility prevalence and treatment-seeking: potential need and demand for infertility medical care. Hum Reprod 2007, 22: 1506-12.

[7] Carlsen E, Giwercman A, Keiding $N$ et al. Evidence for decreasing quality of semen during past 50 years. BMJ1992,30. [Consulté le 30/03/2015]. Disponible à partir d'URL : http://dx.doi.org.www. sndl1.arn.dz/10.1136/bmj.305.6854.609 25

[8] Snick K, Snick T. S., Evers J.L, et al. The spontaneous pregnancy prognosis in untreated subfertile couples: the Walcheren primary care study. Hum Reprod,1997,12 (7):1582-8.

[9] WHO Technical Report Series 894. OBESITY: PREVENTING AND MANAGING THE GLOBAL EPIDEMIC. World Health Organization Geneva 2000.

[10] Couet M,L. Exploration du couple infertile. Zème édition revue et corrigée. Masson. Médecine et biologie de la reproduction Des gamètes à la conception. 2004 (21-51), ISBN: 2-294-01037-X.

[11] Collège National Des Gynécologues Et Obstétriciens Français. Extrait des Mises à jour en Gynécologie Médicale. Trente-deuxièmes journées nationales, Volume 2008Paris.

[12] Pinborg A, Hougaard C.0, Nyboe Andec A, et al. Prospective longitudinal cohort study and cumulative 5-year delivery and adoption rates among 1338 couples initiating infertility treatment. Hum Reprd 2009, 24 (4): 991-999.

[13] Malizia A, Hacker M. R, et Penzias A. S. Cumulative live-birth rates after in vitro fertilization. N Engl J Med. 2009, 360(3):236-43.

[14] Mokdad C, Clavier B, Perdrix A, et al. Facteurs pronostiques en insémination avec sperme de donneur : suivi rétrospectif d'une cohorte de 188 patientes. Gynécologie Obstétrique \& Fertilité 2013, 41(2) : 96-104.

[15] Assistance médicale à la procréation. College National des gynecologuesobstetriciensfrançais (CNGOF) TICEM-UMVF MAJ. 02/07/2004.

[16] Klonoff-Cohen H. S, NatarajanL. The effect of advancing paternal age on pregnancy and live birth rates in couples undergoing in vitro fertilization or gamete intrafallopian transfer. Am J ObstetGynecol 2004; 191:507-514.

[17] AboulgharM.A, Mansour R.T, Al-Inany H.G, et al. Paternal age and outcome of intracytoplasmic sperm injection. Reproductive BioMedicine Online 2007,14(5): 588-592. 
[18] DeLaRochebrochard E,deMouzon J,ThepotF,et al. Over40andincreasedfailuretoconceive:thelessonsofin vitro fertilization in France. Fertil Steril2006, 85:1420-4.

[19] De La RochebrochardE et al. Des hommes médicalement assistés pour procréer : IAD, FIV, ICSI, bilan d'une révolution dans la prise en charge médicale de l'infertilité masculine. In: Population, 58e année, n 4-5, 2003 : 549-586, ISBN : 2-7332-3046-8. [Consulté le 24/12/2013]. Disponible à partir de URL : http://www.persee.fr/web/revues/ home/prescript/article/pop_00324663_2003_num_58_4_7413. 42.

[20] Lintsen AM, Pasker-de Jong PC, de Boer EJ, et al. Effects of subfertility cause, smoking and body weight on the success rate of IVF.Hum Reprod 2005, 20(7):1867-75.

[21] Hassan M.A, Killick S.R.Negative lifestyle is associated with a significant reduction in fecundity.FertilSteril 2004, 81(2): 384-392.

[22]Augood C, Duckitt K, Templeton A. Smoking and female infertility: a systematic review and meta-analysis. Hum Reproduct1998,13(6):1532-1539. [Consulté le 30/05/2018]. Disponible à partir de URL http://humrep.oxfordjournals.org.www.sndl1.arn.dz/ search?author1=C+Augood\&sortspec=date\&submit=Submit»

[23] Hull M.G.R, North K, Taylo H, et al. Delayed conception and active and passive smoking. Fertility and Sterility 2000, 74 (4): 725-733.

[24] Smoking and infertility Practice Committee of the American Society for Reproductive Medicine. FertilSteril 2006;86 Suppl5: S172-S177.

[25] Matalliotakis I, CakmakH ,Arici A et al. Epidemiological factors influencing IVF outcome: Evidence from the Yale IVF program. J of Obstetrics \&Gynaecology 2008,28 (2): 204-208.
[26] Linsten AM et al. Predicting engoing pregnancy chances after IVF and ICSI a national prospective study. Hum Reprod 2007, 22(9): 2455 62 .

[27] BotchanA,HauserR,GamzuR et al. Resultsof6139artificialinseminationcycleswithdonorspermatozoa. HumReprod 2011, 6(11):2298304.

[28] Shibahara H, Hirano Y, Okajima T et al. Establishment of criteria for elective single embryo transfer at day 2 or day 3 by analyzing cases with successful implantation of all embryos transferred. Journal Obstetr and Gync Research 2007, 33 (4):501-505.

[29] Alsalili M, Yuzpe A, Tummon let al.Pregnancy: Cumulative pregnancy rates and pregnancy outcome after in-vitro fertilization: > 5000 cycles at one centre. Hum. Reprod1995, 10(2):470-474.

[30] Farhi J et al. Male factor infertility, low fertilisation rate following ICSI and low number of high- quality embryos.Acta obstetgynecolscand 2008.

[31] Enginsu M. E, Pieters M. H. C, Dumoulin J. C. M et al.Male factor as determinant of in-vitro fertilization outcome.Oxford JournalsHum Reproduct, (8) :1136-1140.

[32] Bostofte E, Serup J, RebbeH. Relation between morphologically abnormal spermatozoa and pregnancies obtained during a twentyyear follow-up period.Int J Androl 1982, 5(4):379-86.

[33] Jouannet P, Ducot B, Feneux D et al. Male factors and the likelihood of pregnancy in infertile couples. I. Study of sperm characteristics. International Journal of Andrology1988, 11(5):379-394. 